\title{
Intensity-based laser distance measurement system using 2D electromagnetic scanning micromirror
}

\author{
Kyoungeun Kim, Jungyeon Hwang and Chang-Hyeon Ji (i)
}

\begin{abstract}
In this research, we present the feasibility testing results of a simple distance measurement system using microfabricated scanning micromirrors. Two different configurations have been tested with different types of micromirrors. In the first configuration, Lissajous scan pattern has been generated with horizontal scan frequency of $715 \mathrm{~Hz}$, and the intensity of the laser beam reflected from the object has been measured with avalanche photodiode to estimate the distance. The position of the beam has been tracked using a separate position sensitive detector. Signals from both sensors are synchronized by eliminating the signal delay, which enables the detection of the distance of a specific point in the 2-dimensional scan pattern. In the simplified configuration, faster scanning micromirror with horizontal scan frequency of $28.8 \mathrm{kHz}$ has been used to increase the resolution and position sensitive detector has been removed from the system by synchronizing the driving current waveform with the avalanche photodiode signals. Distance measurement from 20 to $50 \mathrm{~cm}$ has been demonstrated with the developed system.
\end{abstract}

\section{Introduction}

Recently, technologies related to the optical measurement of 3-dimensional (3D) profile or distance is gaining significant research momentum due to growing needs in various fields of application. LIDAR (light detection and ranging) sensor has become a crucial component for data collection and reconstruction of the 3D space surrounding the autonomous vehicle, and facial or gesture recognition sensors are being actively deployed in hand-held smart devices and automotive vehicles. In general, three different approaches are used for optical distance measurement using the laser beam as a light source, which are TOF (time-of-flight) measurement, triangulation, and intensity measurement methods. The TOF measurement utilizes the time difference between outgoing and incoming laser pulses [1]. Although the long-distance measurement capability with high accuracy makes the technique an attractive choice for applications such as LIDAR systems for autonomous vehicles, complex and costly

\footnotetext{
*Correspondence: cji@ewha.ac.kr

Department of Electronic and Electrical Engineering, Ewha Womans University, Seoul 03760, Republic of Korea
}

architecture for photon-counting and autocorrelation algorithms are required. Despite the low system complexity and fast response, utilization of triangulation is limited as the measurable distance is proportional to the reflected laser beam displacement [2]. Intensity measurement method requires a very simple system architecture, which makes it a reasonable choice for short range distance measurement and motion tracking. Laser sensing display and gesture recognition system from the University of Tokyo are good examples of the intensity-based distance measurement systems [3-5]. As the 3D measurement of distance inherently requires the scanning of laser beam in 2D (2-dimensional) space, motorized scanners are widely used in conventional systems. Recently, various approaches have been proposed to utilize MEMS (microelectromechanical systems) scanners in these applications, which can potentially provide a significant reduction of overall volume and cost of the system $[1,6]$.

In this research, we present the feasibility testing results of a simple distance measurement system using electromagnetic 2D MEMS scanner. In the proposed system, the intensity of the reflected laser beam is measured with APD (avalanche photodiode) 
to estimate the distance and position of the beam is tracked using PSD (position sensitive detector). As shown in Fig. 1, two different types of electromagnetic 2D MEMS scanners with different scan frequency have been tested, and simplified system without the aid of PSD has also been developed [7].

\section{Distance measurement using a 2D low-frequency Lissajous scanner}

Block diagram and optical setup used in the experiment are shown in Fig. 2. Input beam from the laser diode (LD) is incident on the MEMS scanner to generate a 2D Lissajous scan pattern at the image plane. Half of the steered
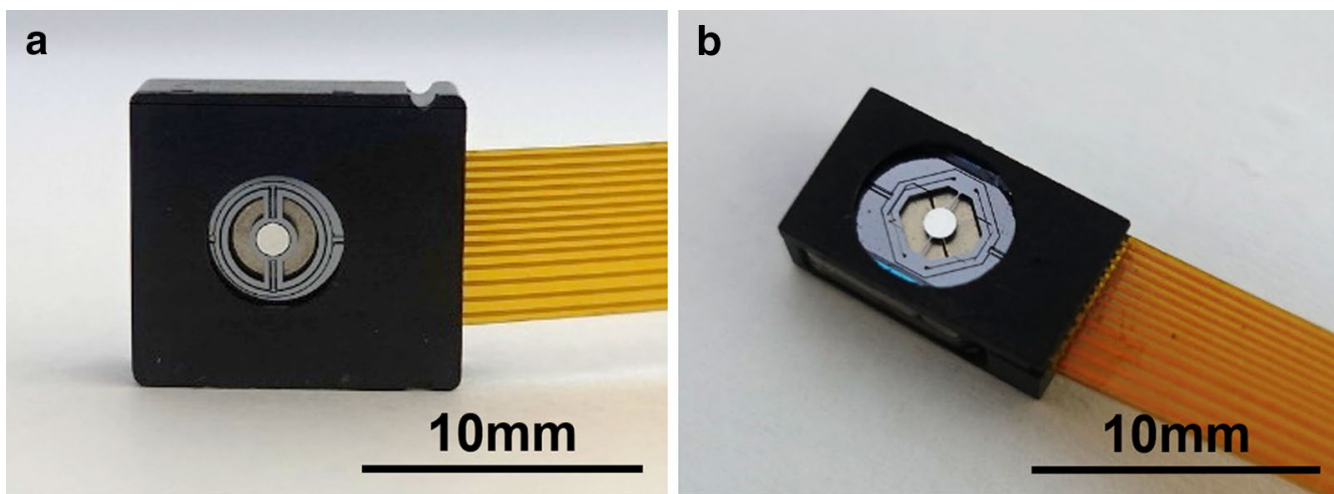

Fig. 1 Electromagnetic 2D scanning micromirrors used in the experiment: a low-frequency scanner, b high-frequency scanner

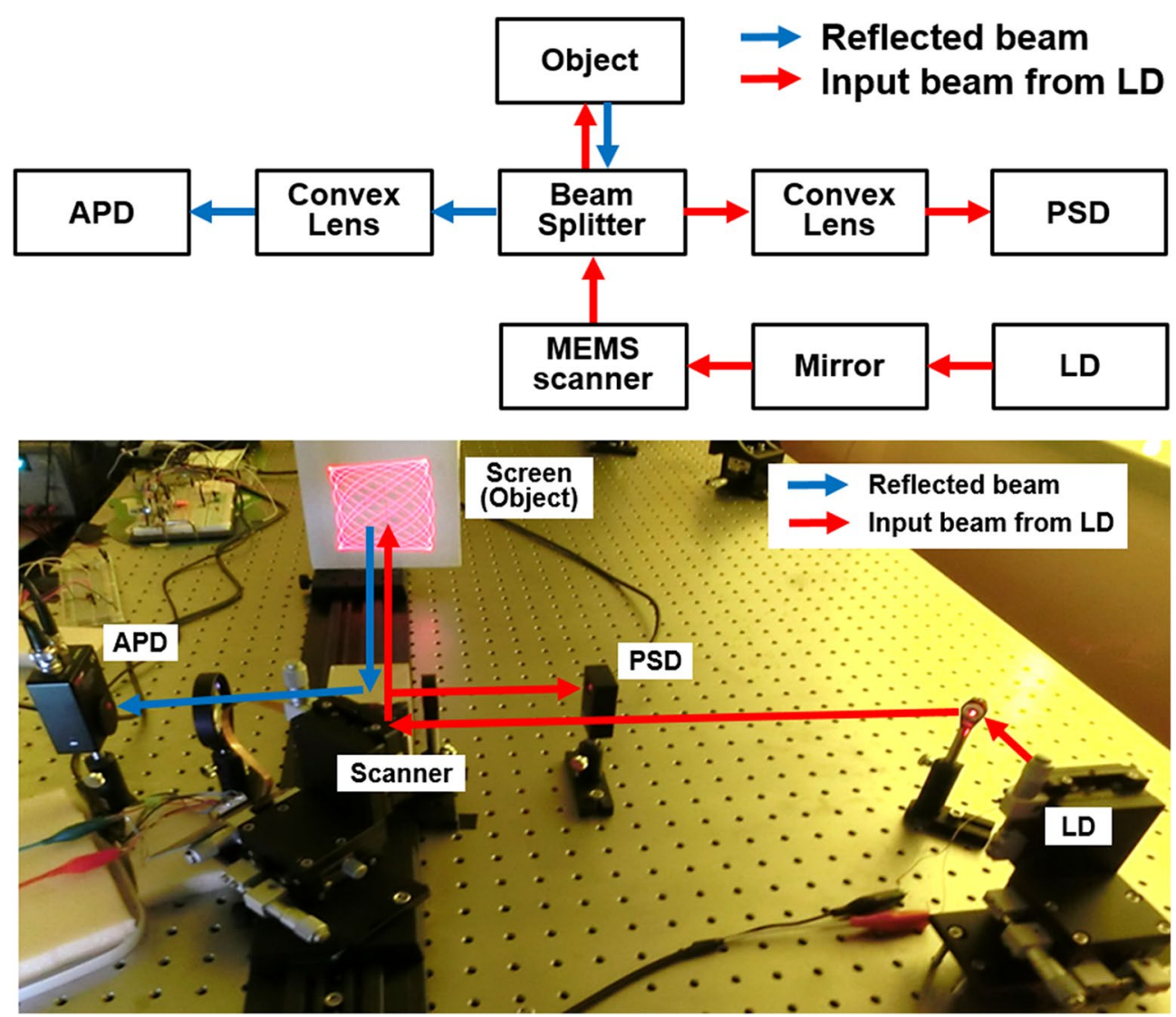

Fig. 2 Block diagram (top) and optical setup (bottom) of the distance measurement system (Lens in front of APD: 50.8 mm-diameter, f60, lens in front of PSD: $25.4 \mathrm{~mm}$-diameter, f 125) 
beam reflected from the scanner is directed towards the PSD sensor which detects the beam displacement to track the beam position on the scan pattern. The beam reflected from the object goes back to the APD via beam splitter where the intensity is measured. A $638 \mathrm{~nm}$ laser diode with $120 \mathrm{~mW}$ output power has been used as the laser source, and an electromagnetic 2D scanner with a mirror diameter of $1.2 \mathrm{~mm}$ and resonance frequency lower than $1 \mathrm{kHz}$ for both scan axes has been used for beam scanning (Fig. 1a). The scanner was originally developed for vector-graphic scanning, but can also be used as a Lissajous scanner by providing sinusoidal input to driving coils for both scan axes [8]. To fully cover the large scan pattern, relatively large optical components have been used including the beam splitter which measured $5 \times 5 \times 5 \mathrm{~cm}^{3}$. A $5.08 \mathrm{~cm}$-diameter bi-convex lens has been placed in front of the APD sensor to transfer the maximum amount of the reflected beam from the object into a single APD sensor (APD130A2, Thorlabs) with $1 \mathrm{~mm}$-diameter. Figure $3 \mathrm{a}, \mathrm{b}$ show the actual Lissajous pattern captured at the image plane and scan pattern reconstructed with PSD sensor (PDQ80A, Thorlabs) signal. Scan frequency along the $x$-axis is $715 \mathrm{~Hz}$ and that of the $y$-axis is $520 \mathrm{~Hz}$. Applied current for the $x$-axis and $y$-axis scan are $96 \mathrm{~mA}$ and $88 \mathrm{~mA}$, respectively. Optical scan angle is approximately $10.5^{\circ}$ for both directions. Discrepancies between the pattern reconstructed with PSD signal and actual scan pattern can be ascribed to the optical misalignment and insufficient resolution of the PSD.

To demonstrate the target object scanning and distance measurement, a small circular mirror with a mount has been utilized as the target. To correctly detect the distance of the target object through laser scanning, signals from APD and PSD sensors have to be synchronized. To identify the possible delay between the sensor signals, a low-frequency input pulse has been applied to the LD, and the output signal from the APD and PSD sensors have been measured. As shown in Fig. 4, the APD sensor had almost no delay, while the PSD sensor had a delay of approximately $0.524 \mathrm{~ms}$. Therefore, after acquiring each signal from the sensors, the delay has to be taken into account for synchronization. Figure 5 shows the typical output waveform of the APD sensor. After eliminating the delay between the two signals, the position of the measured point can be obtained by mapping the signals on the same plane as shown in Fig. 6. Distance data for each measurement point can also be acquired in the form of PSD sensor output voltage (intensity of the beam incident on the PSD). In the experimental setup, the distance between the target object (mirror) and the beam splitter is approximately $37 \mathrm{~cm}$. As the intensity of the laser beam entering the PSD sensor varies depending on the position and reflectivity of the target object, distortion of the scan pattern cannot be avoided, which is evident in the scan pattern shown in Fig. 6b. In addition to the removal of the time delay between the two sensor signals, external triggering is required to maintain the scan pattern and thus the position of the measurement points. As the

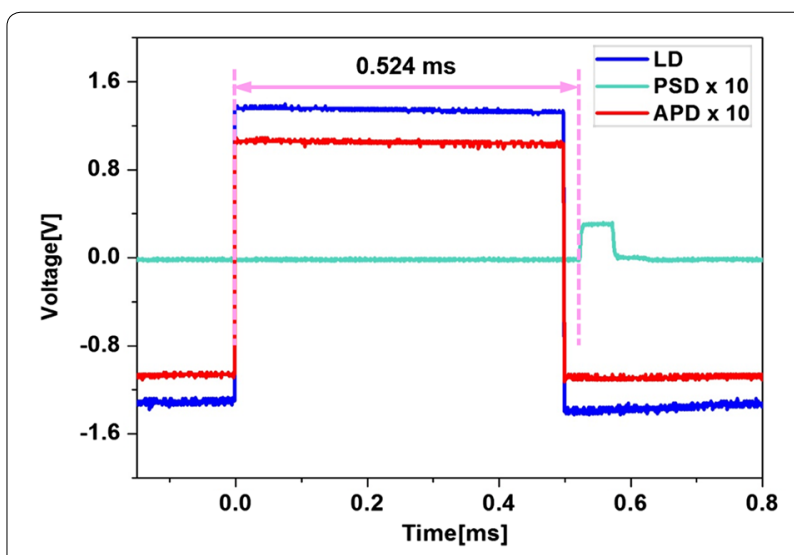

Fig. 4 LD input signal and output signals of the PSD and APD

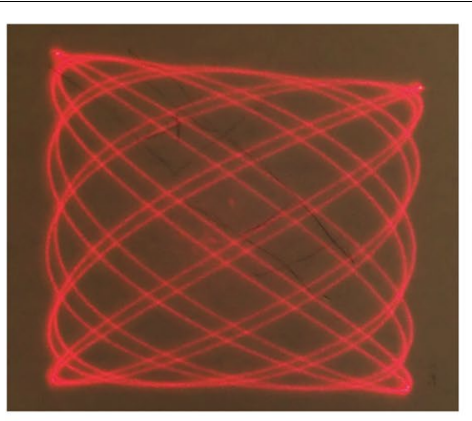

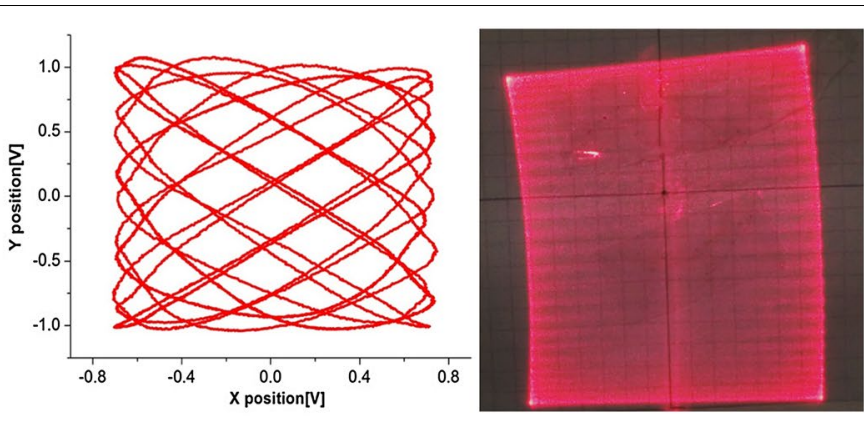

b

C

Fig. 3 a Lissaujous scan pattern (area of the scan region is approximately $8 \times 8 \mathrm{~cm}^{2}$ ), b Lissajous scan pattern reconstructed with PSD sensor signal, c scan pattern generated with $29 \mathrm{kHz}$ scanner (area of the scan region is approximately $7 \times 8 \mathrm{~cm}^{2}$ ) 


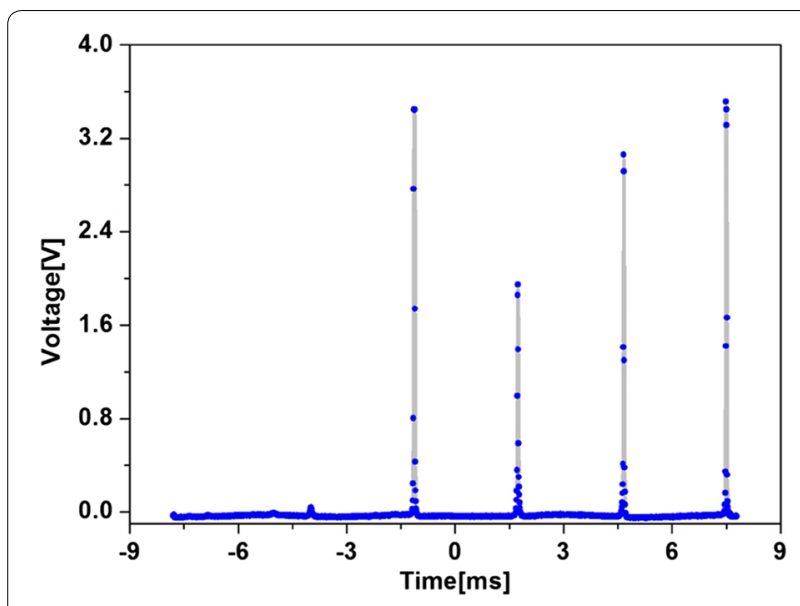

Fig. 5 Output waveform of the APD sensor output from the PSD has two separate signals for $x$ and $y$ scan axes, the frequency of the external triggering signal is adjusted to the greatest common factor, $65 \mathrm{~Hz}$, of the two scan frequencies. After checking out the feasibility of simultaneously measuring the position and intensity of the laser beam reflected from the high reflectivity mirror object, additional experiments have been conducted to confirm whether it is possible to measure the distance of other low reflectivity materials. Figure 7 shows the measurement result of a paper as the object. Results shown in Figs. 6 and 7 verify that the position and distance measurement of $2 \mathrm{D}$ region with various reflectivity is feasible using the proposed method, although the relationship between the measured intensity and distance has to be adjusted depending on the reflectivity of the material.

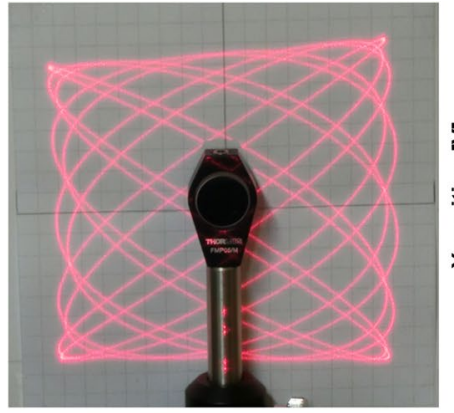

a

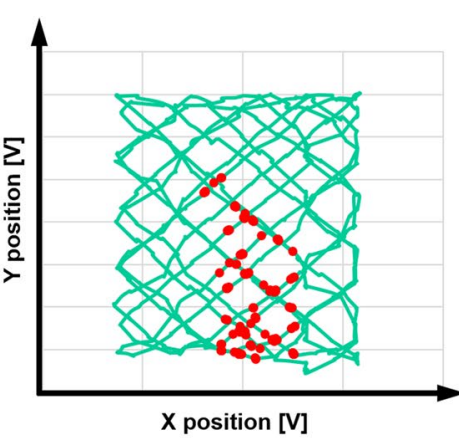

b

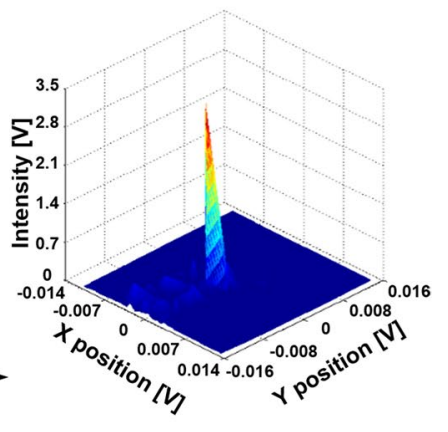

C

Fig. 6 Position and intensity measurement result: a mirror position inside the scan pattern, $\mathbf{b}$ reconstructed scan pattern and intensity measurement points, $\mathbf{c}$ intensity of the reflected beam vs. xy position

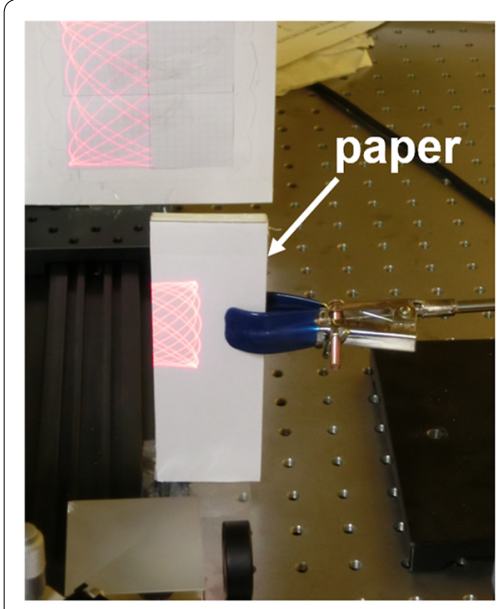

a

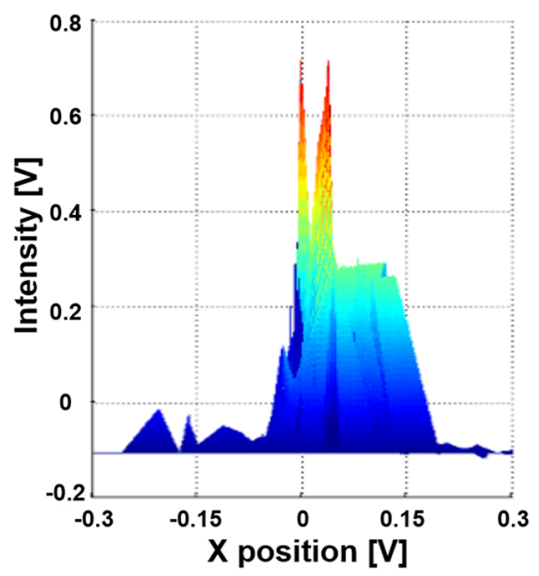

b

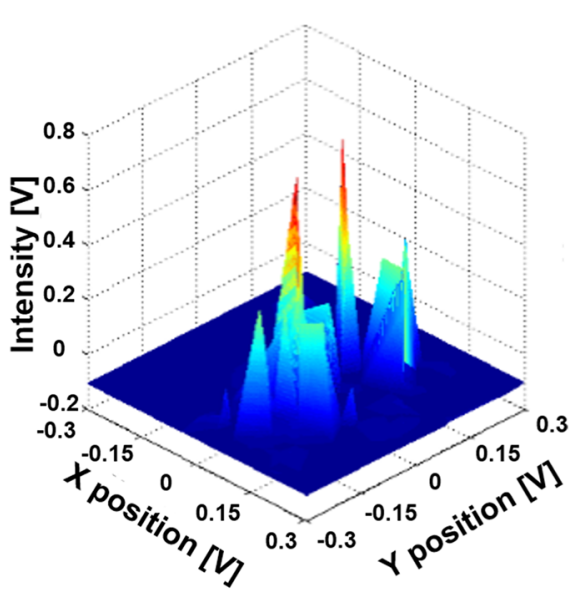

C

Fig. 7 Position and intensity measurement result of a paper located on the right side: a position of the paper, $\mathbf{b}$ intensity of the reflected beam vs. $x$ position, $\mathbf{c}$ intensity of the reflected beam vs. xy position 
However, as shown in the previous results, the intensity measurement results are distributed unevenly due to the sparse scan pattern generated with a relatively slow scanner. Although not demonstrated in this work, high definition and high frame rate Lissajous scan can be achieved by appropriate selection of the scan frequency without having to increase the scan frequency dramatically [9].

\section{Distance measurement using a 2D high-frequency scanner}

To improve the resolution of the system, electromagnetic 2D scanner with a mirror diameter of $1 \mathrm{~mm}$ and horizontal resonance frequency of $28.8 \mathrm{kHz}$ has been tested (Fig. 1b). The device was originally designed for a highdefinition pico projection display system, and horizontal and vertical resonance frequencies are $28,775 \mathrm{~Hz}$ and $493 \mathrm{~Hz}$, respectively $[10,11]$. The operating frequencies are adjusted to generate a scan pattern with the desired area. Used horizontal and vertical scan frequencies are $28,761 \mathrm{~Hz}$ and $495 \mathrm{~Hz}$, respectively. Applied current for the $x$-axis and $y$-axis scan are $68 \mathrm{~mA}$ and $5 \mathrm{~mA}$, respectively. Optical scan angle are approximately $9.2^{\circ}$ for the $x$-axis and $10.5^{\circ}$ for the $y$-axis. Instead of combining the resonance mode actuation for the $x$-axis and forced mode actuation for the $y$-axis, resonance mode actuation is used for both axes for simplicity. To maintain a stable scan range, resonance mode actuation requires continuous track and trace of resonance frequency and scan angle, while forced mode actuation requires a PD (proportional-derivative) control to remove the ringing during vertical scan. As the micromirrors used in the experiment are not equipped with integrated deflection angle sensor, we have utilized resonance mode actuation for both axes without feedback control. For the development of more simple and cost-effective device architecture, PSD is removed and driving current waveform and the APD sensor signal are synchronized by $495 \mathrm{~Hz}$ rising edge triggering signal. As the output from the function generator passes through a voltage follower circuit to drive the 2D scanner, elimination of the time delay between the function generator and the APD sensor signals is required. The measured time delay is $0.857 \mathrm{~ms}$. To compare the position and distance measurement results, previously experimented mirror object is scanned with
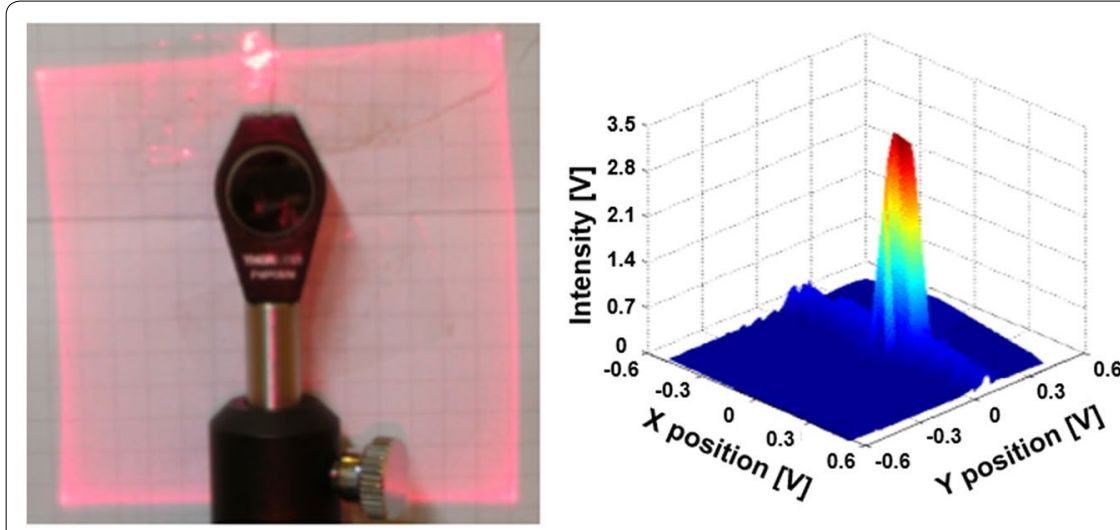

a
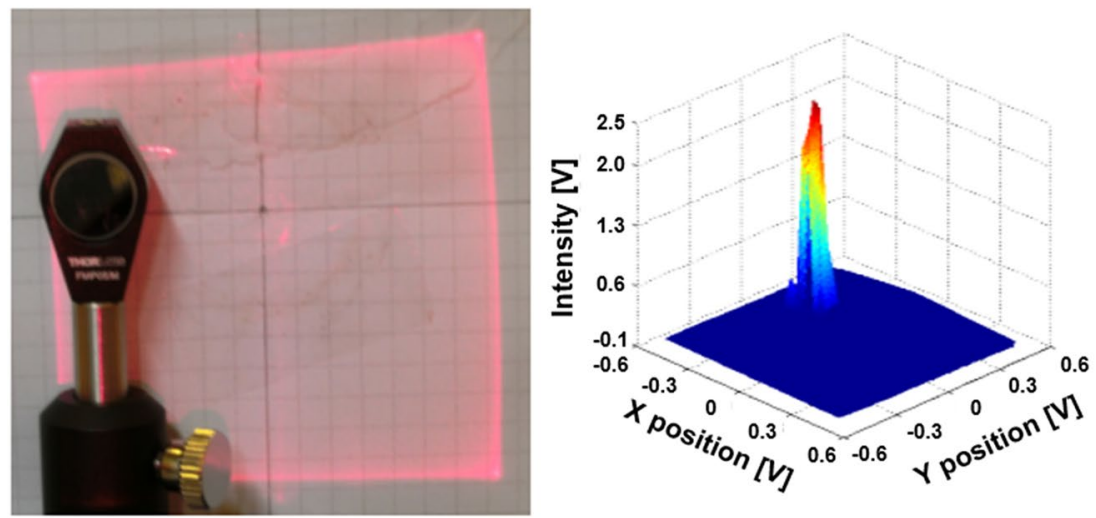

b
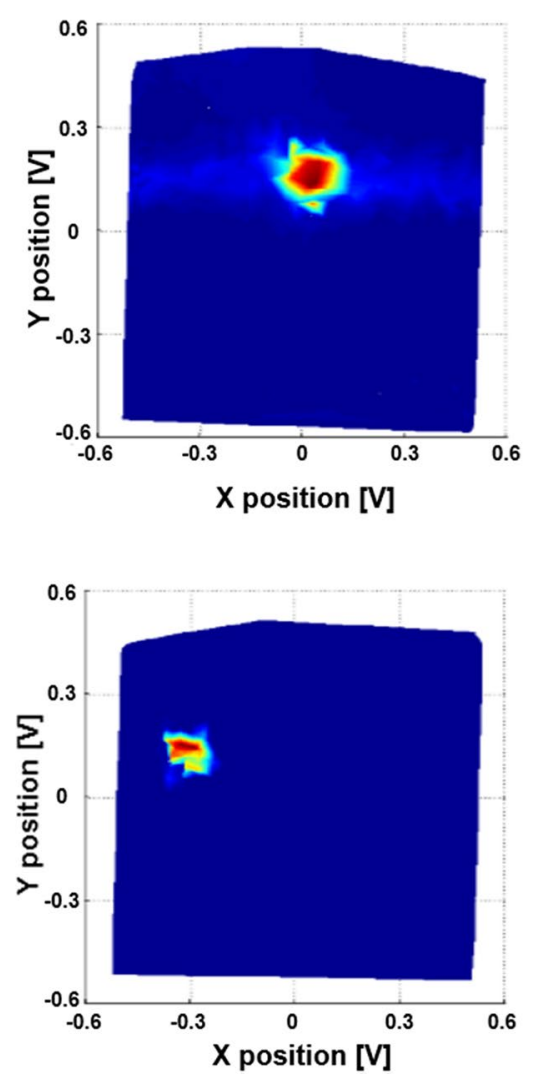

Fig. 8 Position and intensity measurement result using $29 \mathrm{kHz}$ scanner (mirror position inside the scan pattern (left), intensity of the reflected beam vs. xy position (middle), top view of the graph in the middle (right)): a mirror located in the center, $\mathbf{b}$ mirror located on the left side 


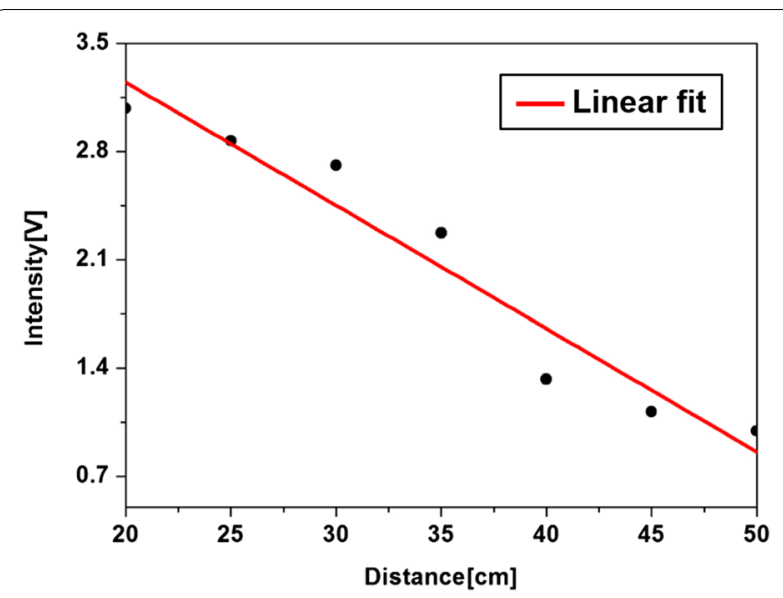

Fig. 9 Measured intensity vs. distance

the high-frequency scanner. As shown in Fig. 8, improved results with more data points have been obtained compared to those achievable with low-frequency scanner. To estimate the actual distance of the object, the intensity of the reflected beam from the object is measured at various distances. The distance of the mirror is adjusted from 20 to $50 \mathrm{~cm}$ using an optical rail with a $5 \mathrm{~cm}$ step. In Fig. 8 , measured data points indicate average values of maximum intensity point for five repeated measurements. According to the results shown in Fig. 9, measurable distance ranges from 20 to $50 \mathrm{~cm}$.

Although the proposed intensity-based distance measurement system cannot provide an accurate estimation of the absolute distance, relative differences in intensity or rough estimate of the distance and position of an object in 2D space can be obtained. Therefore, the location and approximate profile of the object can be found. Also, the distance of the object at the center of the scan pattern matches relatively well with the equation derived with distance measurement experiment. When the intensity versus distance equations are established for objects located at various positions and angles, it is possible to detect the distances with more accuracy. Therefore, through more experiments and optimization of the optics, more advanced intensity-based distance sensor using a 2D MEMS scanner can be developed for motion tracking and gesture recognition.

\section{Conclusion}

Feasibility testing result of an intensity-based distance measurement system using 2D MEMS scanning micromirror has been presented. Two types of electromagnetic MEMS scanners with different scan frequency have been utilized to create 2D scan patterns. The optical setup for receiving the reflected laser beam from the target object has been constructed. An APD sensor has been utilized in measuring the intensity of the reflected beam, and a PSD sensor has been utilized for detecting the reflected beam position and scan pattern. By synchronizing the APD and PSD outputs, the position and distance of the object in the 2D space can be detected. Also, the PSD sensor can be removed from the optical setup by synchronizing the input waveform applied to the scanner and the APD sensor signal. Although dependence on object surface roughness and reflectivity could not be removed, detection of approximate distance and the position of the target object with simple architecture using a single APD sensor has been demonstrated. For the object with known reflectivity and position, the distance can be determined with more accuracy. Although further experimentation would be required to improve the accuracy of measurement, the feasibility of utilizing 2D MEMS scanner in an intensity-based distance measurement application has been verified successfully.

\section{Authors' contributions}

$\mathrm{KK}$ carried out the experiments, and $\mathrm{KK}, \mathrm{JH}$, and $\mathrm{C}-\mathrm{HJ}$ analyzed the experimental results, and drafted the manuscript. All the authors discussed the proposed architecture and experimental results. All the authors read and approved the final manuscript.

\section{Competing interests}

The authors declare that they have no competing interests.

\section{Funding}

This work was supported by Basic Science Research Program through the National Research Foundation of Korea (NRF) funded by the Ministry of Science, ICT \& Future Planning (2017R1 A2B4007830), Basic Science Research Program through the National Research Foundation of Korea (NRF) funded by the Ministry of Education (NRF-2014R1A1A2057721), and by the Center for Integrated Smart Sensors as GFP (CISS-2012M3A6A6054204).

\section{Publisher's Note}

Springer Nature remains neutral with regard to jurisdictional claims in published maps and institutional affiliations.

Received: 6 September 2018 Accepted: 26 November 2018

Published online: 30 November 2018

\section{References}

1. Moss R, Yuan P, Bai X, Quesada E (2012) Low-cost compact MEMS scanning LADAR system for robotic applications. Proc. of SPIE 8379:1-9

2. Berkovic G, Shafir E (2012) Optical methods for distance and displacement measurements. Adv Opt Photonics 4:441-471

3. http://www.k2.t.u-tokyo.ac.jp/perception/SLP/index-e.html. Accessed 30 Nov 2018

4. Cassinelli A, Zerroug A, Ishikawa M (2010) Camera-less smart laser projector. In: Siggraph 2010

5. Perrin S, Cassinelli A, Ishikawa M (2004) Gesture recognition using laserbased tracking system. In: Proceedings of 6th IEEE International Conference on Automatic Face and Gesture Recognition, pp 541-546

6. Niclass C, Ito K, Soga M, Matsubara H, Aoyagi I, Kato S, Kagami M (2012) Design and characterization of a $256 \times 64$-pixel single-photon imager in CMOS a MEMS-based laser scanning time-of-flight sensor. Opt Express 20(11):11863-11881 
7. Kim K (2017) Feasibility study on LIDAR sensor using MEMS scanning micromirror. Dissertation Ewha Womans University

8. Han A, Cho AR, Ju S, Ahn S-H, Bu J-U, Ji C-H (2016) Electromagnetic biaxial vector scanner using radial magnetic field. Opt Express 24(14):15813-15821

9. Hwang K, Seo Y-H, Ahn J, Kim P, Jeong K-H (2017) Frequency selection rule for high definition and high frame rate Lissajous scanning. Sci Rep $7: 14075$
10. Cho AR, Han A, Ju S, Jeong H, Park J-H, Kim I, Bu J-U, Ji C-H (2015) Electromagnetic biaxial microscanner with mechanical amplification at resonance. Opt Express 23(13):16792-16802

11. Ju S, Jeong H, Park J-H, Bu J-U, Ji C-H (2018) Electromagnetic 2D scanning micromirror for high definition laser projection displays. IEEE Photonic Tech Lett. 30(23):2072-2075

\section{Submit your manuscript to a SpringerOpen ${ }^{\circ}$ journal and benefit from:}

- Convenient online submission

- Rigorous peer review

- Open access: articles freely available online

- High visibility within the field

- Retaining the copyright to your article

Submit your next manuscript at $\boldsymbol{\nabla}$ springeropen.com 